\title{
Synthesis and Characterization of Erbium Lithium Niobium Gallium Garnet
}

\author{
O. Toma ${ }^{a}$, L. GheOrGhe ${ }^{a}$ And A.M. Vlaicu ${ }^{b}$ \\ ${ }^{a}$ National Institute for Laser, Plasma and Radiation Physics, 409 Atomistilor Str., Magurele, Jud. Ilfov \\ 077125, Romania \\ ${ }^{b}$ National Institute for Materials Physics, 105 bis Atomistilor Str., Magurele, Jud. Ilfov, Romania \\ The synthesis of a new garnet, $\mathrm{Er}_{3} \mathrm{Li}_{0.275} \mathrm{Nb}_{0.275} \mathrm{Ga}_{4.45} \mathrm{O}_{12}$ (ErLNGG), by solid-state reaction, is reported. \\ A comparison of the fluorescence spectra of $\mathrm{Er}^{3+}$ in ErLNGG, Er-doped (0.1 at.\%) calcium lithium niobium \\ gallium garnet (CLNGG), and $\operatorname{Er}(50$ at.\%):CLNGG is presented. The narrowing of luminescence lines suggests \\ an ordering of the partially disordered CLNGG with the increase of erbium concentration.
}

PACS: $42.70 . \mathrm{Hj}, 78.55 . \mathrm{Hx}$

\section{Introduction}

At present, there is increasing interest in rare-earth-doped laser crystals with inhomogeneously-broadened optical spectra. The rare-earth-doped partially disordered garnets calcium niobium gallium garnet (CNGG) and calcium lithium niobium gallium garnet (CLNGG) are such crystals, having broad spectral bands and mechanical and thermal properties superior to glasses. Their wide absorption bands make them suitable active media for laser-diode-pumped solid-state lasers [1-3], while their wide emission bands can be used to obtain tunable solid-state lasers and ultrashort-pulse lasers [4-6].

Erbium-doped CNGG and CLNGG were studied for the possibility of improving the laser emission on transitions ${ }^{4} I_{11 / 2} \rightarrow{ }^{4} I_{13 / 2}$ and ${ }^{4} I_{13 / 2} \rightarrow{ }^{4} I_{15 / 2}$; to our knowledge, the investigation of the spectroscopic parameters of these crystals was limited up to now to low dopant concentrations $[7,8]$.

In this paper, the solubility limit of $\mathrm{Er}^{3+}$ in CLNGG is found to be 100 at.\%; this value is proved by the synthesis of the erbium lithium niobium gallium garnet (ErLNGG). The synthesized crystalline powder is investigated by X-ray diffraction and optical spectroscopy. The luminescence spectra of ErLNGG are compared with the spectra obtained in $\operatorname{Er}(0.1$ at.\%):CLNGG and $\operatorname{Er}(50$ at.\%):CLNGG.

\section{Experimental}

For the synthesis of the ErLNGG and Er:CLNGG, a solid-state reaction technique was used. A Nabertherm LHT 02/18 furnace was used for the thermal treatments.

The X-ray diffraction analysis was performed using a Bruker diffractometer with $\mathrm{Cu} K_{\alpha_{1}}$ radiation.
The luminescence spectra of the ErLNGG were obtained using a powder sample in a quartz cuvette; the powder was obtained by grinding the synthesized ceramic pellet in an agate mortar with pestle.

For the luminescence of $\operatorname{Er}(0.1 \%)$ :CLNGG, a bulk crystalline sample was used. The $\operatorname{Er}(50 \%)$ :CLNGG sample was also a powder synthesized by solid-state reaction and its luminescence was measured using a quartz cuvette.

The luminescence spectra on the transition $\left({ }^{4} S_{3 / 2},{ }^{2} H 2_{11 / 2}\right) \rightarrow{ }^{4} I_{15 / 2}$ were excited in UV (wavelength range $300-400 \mathrm{~nm}$ ) using a $\mathrm{Xe}-\mathrm{Hg}$ arc lamp with a $\mathrm{CuSO}_{4}$ solution filter and a Schott UG1 glass filter. The luminescence was collected using a concave mirror and focused on the entrance slit of a Horiba Jobin-Yvon $1000 \mathrm{M}$ Series II monochromator. The detector was an EMI S-20 photomultiplier. For the recording of data we used a SR540 optical chopper and a SR830 lock-in amplifier from Stanford Research Systems.

An absorption spectrum of a bulk $\operatorname{Er}(5 \%)$ :CLNGG sample was recorded using a tungsten-halogen lamp included in a similar setup.

\section{Results and discussion}

The stoichiometric composition of the erbium lithium niobium gallium garnet was chosen to correspond to the optimum lithium content of CLNGG [9]: $\mathrm{Er}_{3} \mathrm{Li}_{0.275} \mathrm{Nb}_{0.275} \mathrm{Ga}_{4.45} \mathrm{O}_{12}$. For its synthesis, the following raw materials were used: $\mathrm{Li}_{2} \mathrm{CO}_{3}$ (Serva International), $\mathrm{Nb}_{2} \mathrm{O}_{5}$ (Johnson Matthey Chemicals Ltd., spectrographically standardized), $\mathrm{Ga}_{2} \mathrm{O}_{3}$ (Alfa Aesar, 99.999\%), and $\mathrm{Er}_{2} \mathrm{O}_{3}$ (Aldrich Chem. Co., 99.99+\%). The raw materials were mixed in the right proportions and homogenized using an agate mortar and pestle. The mixture was then pressed in a pellet and kept for $15 \mathrm{~h}$ at $900^{\circ} \mathrm{C}$ to 
decompose the $\mathrm{Li}_{2} \mathrm{CO}_{3}$; afterwards, the pellet was thermally treated for $36 \mathrm{~h}$ at $1300^{\circ} \mathrm{C}$. A polycrystalline ceramic pellet was obtained. Polycrystalline CLNGG ceramics were obtained by the same method, using $\mathrm{CaCO}_{3}$ (Riedel-de Haën, precipitated, puriss.) instead of $\mathrm{Er}_{2} \mathrm{O}_{3}$.

For the synthesis of the Er:CLNGG crystals with concentrations $0.1 \%$ and $5 \%$, the raw materials consisted of CLNGG and ErLNGG, ground and mixed in the right proportion. The mixture was homogenized using the agate mortar and pressed in pellets. The pellets were thermally treated for $36 \mathrm{~h}$ at $1300^{\circ} \mathrm{C}$; after the treatment, they were used for growing Er:CLNGG single crystals by the Czochralski technique.

A similar method was used for the preparation of the $\operatorname{Er}(50 \%)$ :CLNGG sample; after the thermal treatment, the resulting pellet was ground in the agate mortar.

The growth of the Er:CLNGG single crystals was performed by pulling from the melt contained in a platinum crucible of $30 \mathrm{~mm}$ diameter and $30 \mathrm{~mm}$ height, in air. The pulling rate was $2 \mathrm{~mm} / \mathrm{h}$ at a rotation rate of $20 \mathrm{rpm}$. The melting was done by induction heating at $1450^{\circ} \mathrm{C}$. The Er:CLNGG single crystals were grown using a $\langle 111\rangle$-oriented seed obtained from a preliminary platinum wire-grown crystal.

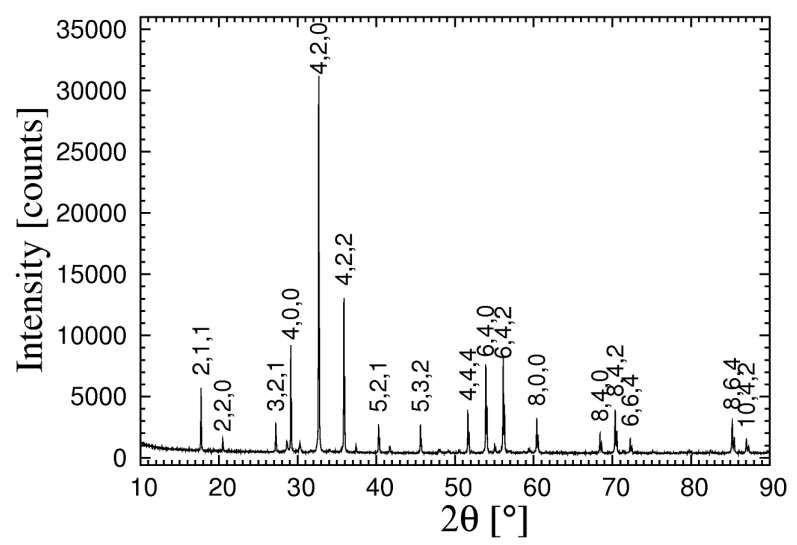

Fig. 1. X-ray diffraction pattern of the ErLNGG powder. The main diffraction peaks are labeled with the corresponding Miller indices.

The X-ray diffraction pattern obtained for the ErLNGG sample is presented in Fig. 1. It proves the garnet structure of the sample and presents no lines corresponding to other crystalline phases. The main diffraction peaks are associated in Fig. 1 to their corresponding Miller indices [10]. The lattice parameter was found $(a=12.2514 \AA)$ at room temperature, smaller than the lattice parameters found for CLNGG $(12.51 \AA)$ and NLNGG $(12.508 \AA$ ) [11]; this is in accord with the ionic radius of $\mathrm{Er}^{3+}$ being smaller than the ionic radii of $\mathrm{Ca}^{2+}$ and $\mathrm{Nd}^{3+}$ in dodecahedral coordination.

The luminescence spectra of the transition $\left({ }^{4} S_{3 / 2},{ }^{2} H 2_{11 / 2}\right) \rightarrow{ }^{4} I_{15 / 2}$ in Er:CLNGG samples of erbium atomic concentrations $0.1 \%, 50 \%$ and $100 \%$ are presented in Figs. 2 and 3. For the ease of comparison, all spectra were normalized to their maximum and the spectrum of the $50 \%$ sample is presented in both figures. A decrease of the linewidths of the spectra is easily observed with the increase of the erbium concentration, denoting a decrease of the disorder of the neighborhood of $\mathrm{Er}^{3+}$. This trend is opposite to the evolution of the linewidths with the increase of dopant concentration observed in an ordered garnet (YAG) and described in [12]. This can be explained if we take into account the different nature of disorder treated in [12]. While in the present work the disorder is an intrinsic characteristic of the CLNGG lattice, in the Er:YAG crystals studied in [12] the disorder is due to the random spatial distribution of the dopant ions in the ordered host lattice.

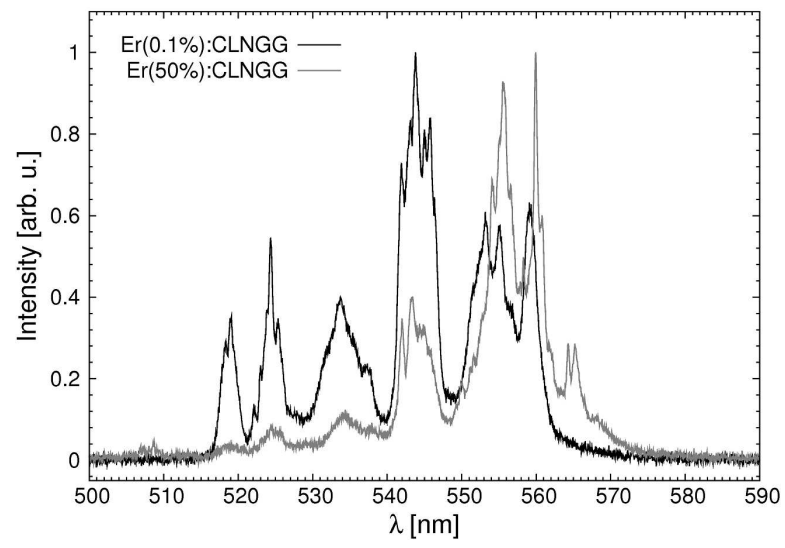

Fig. 2. Normalized luminescence spectra of transition $\left({ }^{4} S_{3 / 2},{ }^{2} H 2_{11 / 2}\right) \rightarrow{ }^{4} I_{15 / 2}$ in $\operatorname{Er}(0.1 \%)$ :CLNGG (black line) and $\operatorname{Er}(50 \%)$ :CLNGG (gray line).

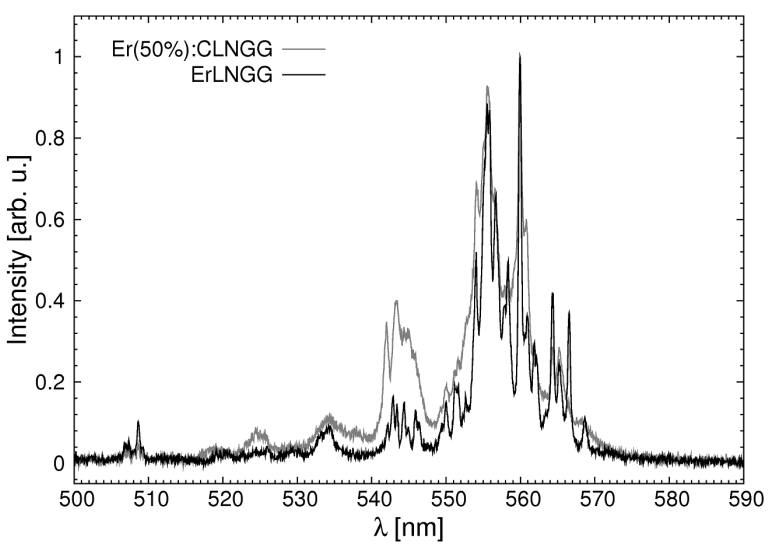

Fig. 3. Normalized luminescence spectra of transition $\left({ }^{4} S_{3 / 2},{ }^{2} H 2_{11 / 2}\right) \rightarrow{ }^{4} I_{15 / 2}$ in $\operatorname{Er}(50 \%)$ : CLNGG (gray line) and ErLNGG (black line).

A change in the intensity distribution of the spectra is also visible: while in $\operatorname{Er}(0.1 \%)$ :CLNGG the most intense line is that located at about $544 \mathrm{~nm}$, for increasing concentrations this line decreases in intensity in comparison with the lines situated at longer wavelengths. The same 
trend is noticed for all luminescence lines at wavelengths shorter than $550 \mathrm{~nm}$. This phenomenon is due to the reabsorption of the luminescence of the ${ }^{2} H 2_{11 / 2}$ level in the sample; reabsorption becomes stronger with the increase of $\mathrm{Er}^{3+}$ concentration in the samples. To support this explanation, we present in Fig. 4 the absorption spectrum of a bulk $\operatorname{Er}(5 \%)$ :CLNGG sample; this spectrum presents intense absorption lines at wavelengths up to $550 \mathrm{~nm}$, in the same spectral range as the luminescence lines that decrease with the increase of $\mathrm{Er}^{3+}$ concentration.

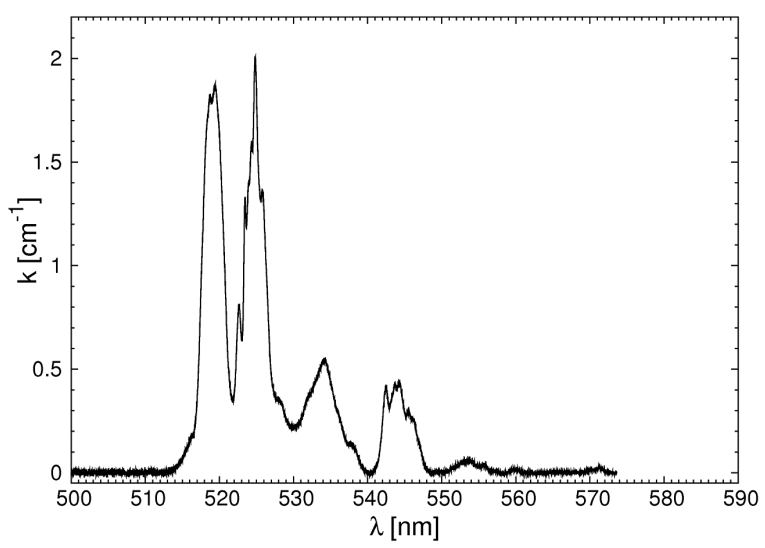

Fig. 4. Absorption spectrum of an $\operatorname{Er}(5 \%)$ :CLNGG sample on transition ${ }^{4} I_{15 / 2} \rightarrow\left({ }^{4} S_{3 / 2},{ }^{2} H 2_{11 / 2}\right)$. The horizontal axis limits were kept the same as in Figs. 2 and 3 for the ease of comparison. The vertical axis represents the absorption coefficient.

\section{Conclusions}

A new crystal, $\mathrm{Er}_{3} \mathrm{Li}_{0.275} \mathrm{Nb}_{0.275} \mathrm{Ga}_{4.45} \mathrm{O}_{12}$, was synthesized by solid-state reaction. Its garnet structure was confirmed by X-ray powder diffraction analysis.

Luminescence spectra obtained on transition ${ }^{4} S_{3 / 2} \rightarrow{ }^{4} I_{15 / 2}$ of $\mathrm{Er}^{3+}$ in samples of Er:CLNGG of various concentrations up to 100 at.\% put into evidence a narrowing of the spectral lines with the increase of erbium concentration. This fact suggests an ordering of the partially disordered CLNGG with the increase of erbium concentration.

\section{Acknowledgments}

The authors are indebted to Dr. S. Georgescu for useful discussions and suggestions.

This work was supported by the post-doctoral research project no. 159/13.08.2010 of the Romanian National Research Council (CNCS).

\section{References}

[1] Y.X. Shi, Q.A. Li, D.X. Zhang, B.H. Feng, Z.G. Zhang, H.J. Zhang, J.Y. Wang, Opt. Commun. 283, 2888 (2010).

[2] H.H. Yu, H.J. Zhang, Z.P. Wang, J.Y. Wang, Y.G. Yu, Z.B. Shi, X.Y. Zhang, M.H. Jiang, Opt. Expr. 17, 19015 (2009).

[3] K.N. He, Z.Y. Wei, D.H. Li, Z.G. Zhang, H.J. Zhang, J.Y. Wang, C.Q. Gao, Opt. Expr. 17, 19292 (2009).

[4] G.Q. Xie, L.J. Qian, P. Yuan, D.Y. Tang, W.D. Tan, H.H. Yu, H.J. Zhang, J.Y. Wang, Laser Phys. Lett. 7, 483 (2010).

[5] A. Schmidt, U. Griebner, H.J. Zhang, J.Y. Wang, M.H. Jiang, J.H. Liu, V. Petrov, Opt. Commun. 283, 567 (2010).

[6] G.Q. Xie, D.Y. Tang, W.D. Tan, H. Luo, H.J. Zhang, H.H. Yu, J.Y. Wang, Opt. Lett. 34, 103 (2009).

[7] A.V. Malov, P.A. Ryabochkina, A.V. Popov, E.V. Bol'shakova, Quantum Electron. 40, 377 (2010).

[8] Yu.K. Voronko, S.B. Gessen, N.A. Eskov, V.V. Osiko, A.A. Sobol, S.N. Ushakov, L.I. Tzimbal, Russ. J. Quantum Electron. (Kvantovaia Elektronika) 17, 721 (1990).

[9] Y.M. Yu, V.I. Chani, K. Shimamura, T. Fukuda, J. Crystal Growth 171, 463 (1997).

[10] E.L. Dukhovskaya, Y.G. Saksonov, A.G. Titova, Izv. Akad. Nauk SSSR, Neorg. Mater. 9, 809 (1973).

[11] L. Gheorghe, M. Petrache, V. Lupei, J. Crystal Growth 220, 121 (2000).

[12] N.I. Agladze, H.S. Bagdasarov, E.A. Vinogradov, V.I. Zhekov, T.M. Murina, M.N. Popova, E.A. Fedorov, Kristallografiya 33, 912 (1988). 